\title{
Prevalence and Predictor of Nonalcoholic Steatohepatitis (NASH) in Nonalcoholic Fatty Liver Disease (NAFLD)
}

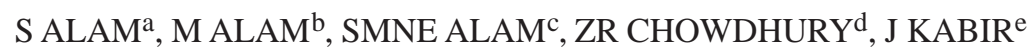

\begin{abstract}
Summary:
Fatty liver is a common cause of chronic liver disease in developed as well as developing countries. We have designed this study to estimate the prevalence and predictors for non alcoholic steatohepatitis (NASH) in non alcoholic fatty liver disease (NAFLD). We have included 493 patients with sonographic evidence of fatty change in liver and 177 of them had done liver biopsy for histopathological study. Other causes of liver disease and alcohol consumption were excluded. Metabolic syndrome and biochemical and anthropometric evaluation was done. Females were predominating 250 (57.0\%). Centrally obese 422 (96.2 \%) was more than over all obesity330 (75.1\%). NASH was absent in 10 (5.6\%) cases and diagnostic of NASH was 75
\end{abstract}

Introduction:

Nonalcoholic fatty liver disease (NAFLD) is a clinicohistopathological entity with histological features that resemble alcohol-induced liver injury. By definition, occurs in patients with little or no history of alcohol consumption ${ }^{1}$. NAFLD is the most common liver disease in western countries, affecting $20-30 \%$ of the general population ${ }^{2,3}$. It encompasses a histological spectrum that ranges from fat accumulation in hepatocytes without concomitant inflammation or fibrosis (simple hepatic steatosis) to hepatic steatosis with a necro-inflammatory component (steatohepatitis)

a. Dr. Shahinul Alam, Associate Professor, Department of Hepatology, Bangabandhu Sheikh Mujib Medical University, Shahbag, Dhaka, Postal Code-1000.Bangladesh.

b. Dr. Mahabubul Alam, Medical Officer. Department of Hepatology, Bangabandhu Sheikh Mujib Medical University, Shahbag, Dhaka, Bangladesh.

c. Dr. Sheikh Mohammad Noor-E-Alam, Resident, Department of Hepatology, BangabandhuSheikhMujibMedicalUniversity, Shahbag, Dhaka, Bangladesh.

d. Dr. ZiaurRahmanChowdhury, Research Assistant, Department of Hepatology, Bangabandhu Sheikh Mujib Medical University, Shahbag, Dhaka, Bangladesh.

e. Dr. Jahangir Kabir, Resident, Department of Hepatology, Bangabandhu Sheikh Mujib Medical University, Shahbag, Dhaka, Bangladesh.

Address of Correspondence: Dr. Shahinul Alam, Associate Professor, Department of Hepatology, Bangabandhu Sheikh Mujib Medical University, Shahbag, Dhaka, Postal Code-1000.Bangladesh. email: shahinul67@yahoo.com

Received: 28 March, 2013

Accepted: 10 December, 2013
(42.4\%).Presence of diabetes could significantly ( $p=0.001)$ predicted NASH. Age, sex, BMI, waist circumference, Serum HDL,triglyceride, insulin resistance index, hypertension, metabolic syndrome could not predict NASH. Serum GGT level was significantly $(p=0.05)$ higher in NASHwith a sensitivity of $45 \%$ and specificity of $68 \%$ only. Serum ALT and AST level could not detect NASH. Females were predominant sufferer of NAFLD in Bangladesh. Prevalence of NASH was much higher42.4\%. Diabetes was the main predictor of NASH. GGT was the only biochemical indicator of NASH. We recommend liver biopsy in NAFLD with diabetes and raised GGT.

(J Banagladesh Coll Phys Surg 2014; 32: 71-77)

that may or may not have associated fibrosis. The latter condition, referred to as nonalcoholic steatohepatitis (NASH), may progress to cirrhosis in up to $20 \%$ of patients ${ }^{4}$. Reports have also suggested that the prevalence of NAFLD among Asian Indians is comparable to that seen in the West ${ }^{5,6}$. Average age for NASH patients is 40-50 years and for NASH-related cirrhosis it is $50-60$ years. NASH probably causes around $80 \%$ of cases of cryptogenic cirrhosis which accounts for $10-20 \%$ of all cirrhosis and progresses to advanced fibrosis in 32 to $37 \%$ of patients ${ }^{7}$.

In parallel with the epidemic of obesity and metabolic syndrome worldwide, the prevalence of NAFLD in Asian countries has increased rapidly with a trend to younger patients during the last two decades. The prevalence of NAFLD was about $15 \%$ in adults in Shanghai and Hong Kong ${ }^{8}$. NAFLD has been associated with insulin resistance and hyperinsulinaemia, even in lean subjects with normal glucose tolerance ${ }^{9}$. Diabetes mellitus may be an independent predictor of NASH, including cirrhosis and hepatocellular carcinoma ${ }^{10}$. NAFLD is now recognized as the hepatic component of the metabolic syndrome, which includes hyperlipidemia, glucose intolerance, obesity, and systemic hypertension. Predictors of NASH increase with the number of components of the metabolic syndrome ${ }^{11}$.The contrasting clinical course of NASH versus non NASH fatty liver (NNFL) indicates that these 
two conditions diverge early in the course of NAFLD although some patients probably transition from NNFL to NASH. Progression to cirrhosis is usually preceded by longstanding histological NASH and is infrequent in NNFL. Longitudinal studies with serial biopsies have shown that about one-third of NASH patients develop advanced fibrosis (stage 3 or 4 fibrosis) over 5-10 years from the time of the initial diagnosis ${ }^{12}$. Although usually relatively slow, progression to cirrhosis can occur in as little as 2-3 years. NASH is a common cause of 'cryptogenic' cirrhosis, which accounts for $10-20 \%$ of all cirrhosis ${ }^{13}$. Among patients diagnosed with NASH-related cirrhosis, the risk of developing a major complication of portal hypertension is 17, 23 and 52\% at 1,3 and 10 years, respectively. Among patients with early stage NASH, overall mortality over 10-15 years is about $10-12 \%$, being significantly higher in NASH versus NNFL, compared to the general population. The risk of developing decompensated cirrhosis is $5-10 \%$ and for hepatocellular cancer it is $1-2 \%$. There is a tenfold risk of cirrhosis relative to the general population ${ }^{14}$.

A complete diagnosis of fatty liver disease ideally should define the histology, including the stage and grade of the disease as well as its etiology. In Bangladesh NAFLD is never been or insufficiently addressed in the field of medical research and practice. NASH is a potentially dangerous condition which requires medical intervention. The prevalence of NASH and potential risk factors for it is not yet explored here. We have designed this study protocol to estimate the prevalence of NASH in NAFLD and predictor of NASH in the perspective of Bangladeshwhich will be helpful future scientific knowledge and intervention.

\section{Materials and Methods:}

\section{Study population:}

We have included initially 439 patients at outpatient department of Hepatology in the University Hospital during the period of March 2010- December 2012 for fatty filtration in liver with ultrasonography. Exclusion criteria consisted of significant alcohol abuse $(<20 \mathrm{~g}$ daily), evidence of hepatitis $\mathrm{B}$ and $\mathrm{C}$ and of drug induced fatty liver and other specific liver diseases: Hemochromatosis, Wilson's disease or autoimmune liver disease. These patients underwent clinical evaluation, anthropometric measurements, and blood tests. Liver biopsy was done after randomization in 190 patients but 4 biopsy samples were inadequate to comment for histopathology 4 patients withdrawn themselves from the study. The study was approved by the Institutional Review Board and all individuals provided written informed consent prior to enrollment in the study. Metabolic syndrome was defined according to Asian criteria, ${ }^{[15]}$ and three of the five listed criteria were considered: waist circumference (WC) e"80 cm for women and e"90 cm for men, serum triglyceride $\geq 150 \mathrm{mg} / \mathrm{dl}$ (1.7 mmol/l), serum HDL cholesterol <50 $\mathrm{mg} / \mathrm{dl}$ (1.3 mmol/l) for women and <40 mg/dl ( $1 \mathrm{mmol} /$ l) for men, elevated blood pressure (systolic blood pressure $\geq 130$ and or diastolic blood pressure $\geq 85$ $\mathrm{mmHg}$ or drug treatment for hypertension) and plasma glucose concentration e” $100 \mathrm{mg} / \mathrm{dl}$ (5.6 mmol/l) or drug treatment for diabetes.

\section{Clinical and Biochemical evaluation:}

All the patients were clinically evaluated: Blood pressure, Body mass index (BMI) and waist circumference was recorded for every patient. Liver function tests were performed prior to the liver biopsy. Blood samples were obtained under fasting conditions and the following tests were performed using standard laboratory methods: alanine aminotransferase (ALT), aspartate aminotransferase (AST), alkaline phosphatase, Gamma glutamyltranspeptidase (GGT) international normalized ratio (INR), blood glucose fasting and 2 hours after breakfast, lipid profile, Insulin level was assessed using the method of indirect chemiluminescence (MEIA). Insulin resistance was calculated according to the HOMA index (Homeostatic Metabolic Assessment).

\section{Histological assessment}

Liver biopsy specimens of 182 were analyzed by pathologist blinded to the patients' clinical and biochemical results. Histopathology was done in the department of Pathology BSMMU. The diagnosis of NASH was based on the Brunt et al criteria, ${ }^{[16]}$ modified by Kleiner et $\mathrm{al}^{17}$. In this scoring system, the degree of disease activity in NAFLD was evaluated using the NAFLD Activity Score (NAS), which was calculated as the unweighted sum of the scores for steatosis (0-3), lobular inflammation (0-3), and hepatocyte ballooning (0-2) and thus ranged from 0 to 8 . A NAS of 5 or more was diagnosed as "definitive NASH”, NAS of 2 or 
less as “non-NASH,” and 3 or 4 as "borderline NASH.” Other than NASH, was considered as NNFL. Hepatic fibrosis staging was as follows: $0=$ no fibrosis; 1 = zone 3 fibrosis only; 2 = zone 3 and portal/periportal fibrosis; 3 = bridging fibrosis; and 4 = cirrhosis.

\section{Statistical analysis}

Results are presented as mean \pm standard deviation (SD) for quantitative data and as numbers or percentages for categorical or qualitative data. Statistical differences in quantitative data were determined using t test or one way Anova test. Qualitative data were compared using the $\mathrm{x}^{2}$ test. Multivariate regression analysis was done to explore the strongest predictor of NASH including the variables with significance in univariate analysis. For all tests, significance was achieved at $\mathrm{p}<0.05$.

\section{Results:}

Patient Characteristics:

Total of 439 patients wereincluded in this study. Females were 250 (57.0 \%) and males were 189 (43.0 \%). Mean age of the sample was $40.8 \pm 10.2$ years. Most of the population was house wife 217 (50.3\%), others were service holder 84 (19.5\%), business man 69 (16.0\%) and students were 59 (13.7\%). Hypertension and diabetes were prevailing in 83 (18.8\%) and 74 (16.8\%) respectively but metabolic syndrome was 188 (42.9\%). Triglyceride was high in 320 (72.8\%). BMI was normal in 51 (11.7\%), over weight 58 (13.2 \%), Obese I 237 (53.9\%) and obese II 93 (21.2 \%) according to criteria for Asian ${ }^{18}$. Most of the patients were centrally obese 422 (96.2 \%) having waist circumference above normal. ALT, AST and GGT level were $54.1 \pm 54.4$, 45.1 \pm 51.8 and $46.6 \pm 33.7 \mathrm{u} / \mathrm{l}$ respectively. Insulin resistance index were higher than normal in 218 (49.6\%).

Histological Changes: Histopathological reports of 182 patients were available but 5 of them did not have fatty change on microscopy. We have included 177 patients for further analysis. There was no significant difference between biopsied and non- biopsied patient regarding clinical, anthropometric and biochemical variables. Steatosis of $<33 \%$ was 73(41.2\%), $33-66 \%$ was 82 $(46.4 \%)$ and $>66 \%$ was $22(12.4 \%)$. Lobular inflammation was absent in 10 (5.6\%), mild in 93 (52.5 \%), moderate in 70 (39.5 \%) and severe in 4 (2.3\%). Ballooning was absent in 5 (2.8\%), few ballooning in 138 (78.0 \%) and prominent ballooning in 34 (19.2\%) (Figure I). No fibrosis was seen in 28 (15.8\%), stage I in 94 (53.3\%), stage II in 40 (22.5\%) and stage III in 15 (8.3\%). None had stage IV fibrosis(Table I).
According to NAS scoring system NASH was absent in 10 (5.6\%) cases, borderline NASH was 92 (52.6\%) and diagnostic of NASH was 75 (42.4\%). So NNFL was 102 (57.6\%) and NASH was 75 (42.4\%).

\section{Predictors of NASH:}

Prevalence of NASH in NAFLD was 75 (42.4\%). There were no significant difference of age, BMI, waist circumference, Serum HDL and triglyceride level, insulin resistance index,sex, hypertension, metabolic syndrome did not differed in NASH and Non NASH. Mean age, BMI and waist circumference was similar in NNFL and NASH patients. Mean triglyceride was higher in NASH and mean HDL was lower in NASH but could not establish statistically significant value. Presence of diabetes could significantly $(\mathrm{p}=0.001$ ) differentiate NASH from NNFL. Serum ALT and AST level could not detect NASH in NAFLD. But serum GGT level was significantly $(\mathrm{p}=0.05)$ higher in NASH than that of NNFL (Table II). GGT level for NASH was (51.7 \pm 32.8) U/L and for NNFL was (40.4 \pm 22.6$) \mathrm{U} / \mathrm{L}$. Multivariate regression analysis also explore that presence of diabetes could influence the development of NASH $(p=0.04)$ and GGT could differentiate NASH from NNFL ( $p=0.01)$ (table III). But area under the curve is $59.3 \%$ for GGT to differentiate NASH, with a sensitivity of $45 \%$ and specificity of $68 \%$ only for 44.5 U/L (Figure II).

\section{Table-I}

Histopathological features of biopsied patients

\begin{tabular}{lcc} 
Variable & Number & Percent \\
\hline Lobular inflammation & & \\
Absent & 10 & 5.6 \\
Mild & 93 & 52.5 \\
Moderate & 70 & 39.5 \\
Severe & 4 & 2.3 \\
Ballooning & & \\
Absent & 5 & 2.8 \\
Few & 138 & 78.0 \\
Prominent & 34 & 19.2 \\
Fibrosis & & \\
Absent & 28 & 15.8 \\
Stage I & 94 & 53.3 \\
Stage II & 40 & 22.5 \\
Stage III & 15 & 8.3 \\
NASH & 75 & 42.4 \\
\hline
\end{tabular}


Table-II

Clinical, anthropometric and biochemical differences of NNFL and NASH

\begin{tabular}{|c|c|c|c|}
\hline Variable & $\begin{array}{l}\text { NNFL } \\
\mathrm{N}=102 \\
\end{array}$ & $\begin{array}{c}\text { NASH } \\
\mathrm{N}=75 \\
\end{array}$ & Pvalue \\
\hline Age (yr)Mean \pm SD & $39.3 \pm 9.4$ & $41.0 \pm 9.7$ & 0.24 \\
\hline Sex: Male/ female & $42 / 60$ & $31 / 44$ & 1.00 \\
\hline Body Mass Index $\left(\mathrm{Kg} / \mathrm{m}^{2}\right)$ & $27.8 \pm 3.9$ & $27.8 \pm 4.6$ & 0.998 \\
\hline Waistcircumference in cm Male & $93.0 \pm 5.5$ & $93.0 \pm 9.8$ & 0.081 \\
\hline Female & $95.8 \pm 9.9$ & $95.6 \pm 11.0$ & 0.927 \\
\hline HDL in mg/dl Male & $36.3 \pm 8.9$ & $34.2 \pm 6.5$ & 0.337 \\
\hline Female & $39.8 \pm 10.3$ & $39.2 \pm 10.3$ & 0.801 \\
\hline Serum Triglyceride mg/dl & $225.2 \pm 165.8$ & $239.8 \pm 111.6$ & 0.509 \\
\hline Insulin Resistance Index & $1.8 \pm 1.3$ & $1.5 \pm 0.7$ & 0.337 \\
\hline Diabetes Present / Absent & 13/86 & $25 / 48$ & 0.001 \\
\hline Hypertension Present / Absent & $17 / 65$ & $17 / 48$ & 0.555 \\
\hline Metabolic SyndromePresent/ Absent & $41 / 41$ & 39/32 & 0.328 \\
\hline ALT U/L & $56.9 \pm 38.8$ & $56.3 \pm 31.8$ & 0.603 \\
\hline AST U/L & $46.9 \pm 63.7$ & $46.1 \pm 22.2$ & 0.916 \\
\hline GGT U/L & $40.4 \pm 22.6$ & $51.7 \pm 32.8$ & 0.05 \\
\hline
\end{tabular}

NASH; Non alcoholic steatohepatitis, NNFL; Non nash fatty liver

Table-III

\begin{tabular}{|c|c|c|c|c|c|}
\hline \multicolumn{6}{|c|}{ Multivariate regression analysis for variable detecting NASH } \\
\hline \multirow[t]{2}{*}{ Model } & \multicolumn{2}{|c|}{ Unstandardized Coefficients } & \multirow{2}{*}{$\begin{array}{c}\text { Standardized Coefficients } \\
\text { Beta } \\
\end{array}$} & \multirow[t]{2}{*}{$\mathrm{t}$} & \multirow[t]{2}{*}{ Sig } \\
\hline & $\mathrm{B}$ & Std. Error & & & \\
\hline (Constant) & 1.247 & .517 & & 2.411 & .018 \\
\hline BMI & .014 & .018 & .124 & .780 & .438 \\
\hline Diabetes & .260 & .125 & .227 & 2.084 & .040 \\
\hline Serum Triglyceride & .000 & .000 & -.105 & -.919 & .361 \\
\hline GGT & .005 & .002 & .289 & 2.473 & .015 \\
\hline Waist Circumference & -.004 & .008 & -.077 & -.491 & .624 \\
\hline
\end{tabular}

a. Dependent Variable: nash and nnfl

NASH; Non alcoholic steatohepatitis, NNFL; Non nash fatty liver

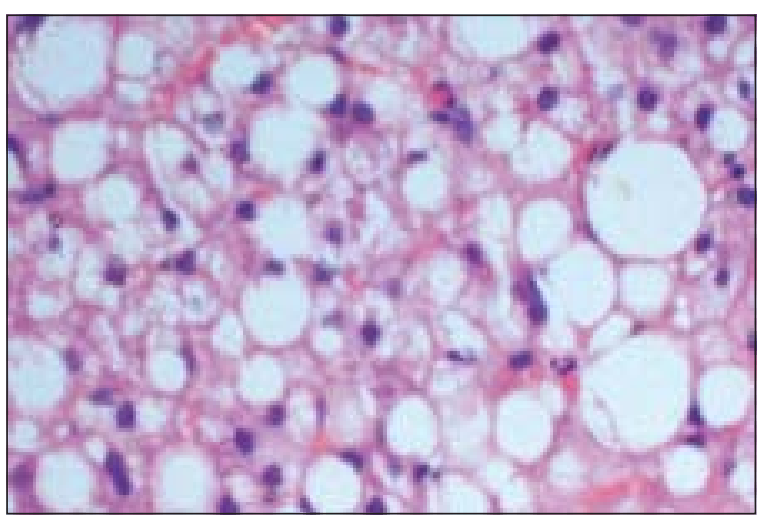

Fig.-1: Microscopic feature of Nonalcoholic steatohepatitis:steatosis and ballooning degeneration.

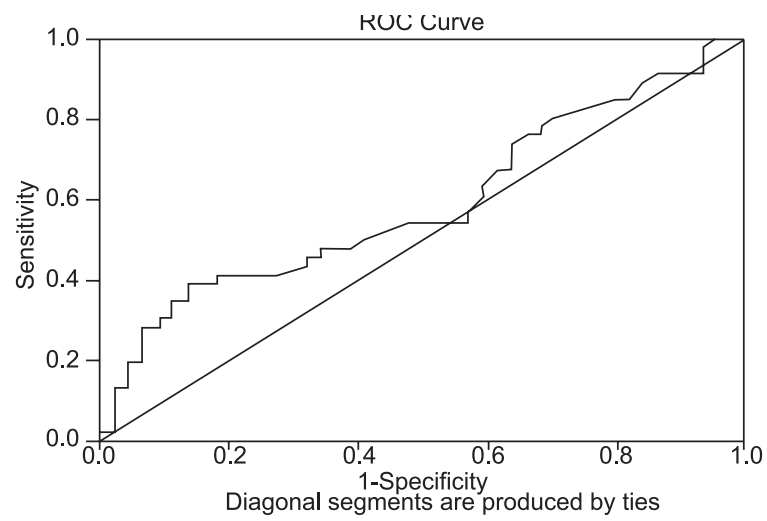

Fig.-2: Receiver Operating Characteristic curve for GGT to differentiate NASH from NNFL. 


\section{Discussion:}

This study is the largest series from Bangladesh on NAFLD. Report of biopsy proven NASH and NNFL is also rare. University hospital is a tertiary care "center of excellence" hospital only and patients are referred from whole over the country. So this study may be the representative of prevalence of NASH in NAFLD of the country. Population based prevalence of NAFLD was not yet done in Bangladesh. Most of our NAFLD patients are of 30 to 50 years; this is similar to several reports from Asia ${ }^{6,19-20}$. But age could not influence the development of NASH. Female preponderance in NAFLD is dissimilar from reports from developed counties. Many recent studies have reported that male gender is a risk factor for fatty liver disease ${ }^{21}$. For example, in a study of 26,527 subjects undergoing medical checkups; the prevalence of NAFLD was 31\% in men and $16 \%$ in women ${ }^{22}$. This female preponderance 250 (57.0\%) in our study may be the social conservative attitude which bounded most of our ladies to stay home for house hold activities without job leading to sedentary life style. Similar female preponderance was observed in one population studies from India ${ }^{23}$. But in accordance with previous studies sex did not influenced the development of NASH in NAFLD 22 .

Centrally obese was 422 (96.2 \%) outnumbered the overall obesity 330 (75.1\%). The prevalence of NAFLD was increased according to the increase of BMI or abdominal circumference reported from Japan ${ }^{24}$. But other report concluded that waist circumference is an independent predictor of advance histological changes in NAFLD than $\mathrm{BMI}^{25,26}$. But waist circumference was similar in NASH and NNFL in our series. It could be explained by that waist circumference indicate visceral obesity but no influence on pathogenesis of NASH at the stage of $2^{\text {nd }}$ hit. Hypertriglyceridemia was very common 320 (72.8 \%) in this study with no difference between NASH and NNFL. TG was long been considered as major factor in the development of NAFLD, ${ }^{5-8}$ but there is mounting evidence that such non-TG lipid molecules are implicated in the pathogenesis of NASH by the process of lipotoxicity. Conversely, formation of TG may actually be a cytoprotective mechanism in liver ${ }^{27,28}$. Our study revealed similar role of TG in NAFLD.

Our study explored that prevalence of NASH was 75 (42.4\%) in NAFLD which is much higher. It is alarming for the country like Bangladesh. It was neither addressed previously nor considered anyway. In previous review, NAFLD wasfoundhighly prevalent (15\% to $45 \%)$ in modern societies, only $10 \%$ to $25 \%$ of cases develop $\mathrm{NASH}$, hepatic fibrosis leading to cirrhosis, end-stage liver disease or hepatocellular carcinoma ${ }^{29}$.In other studies prevalence of NASH was 10 to $30 \%$ in NAFLD 30 and it is less in Asian than that of European ${ }^{31.32}$. We were unbiased in selecting patient for liver biopsy and it was irrespective of clinical, biochemical and anthropometric status of the study population. So it is the representative of prevailing situation in the society. This finding warrants further extensive study on prevalence of NASH in Bangladesh and awareness of clinician is essential to diagnose NASH and to advice possible intervention as early as possible.

Presence of diabetes signified the presence of NASH in our study population $(\mathrm{p}=.001)$. Metabolic syndrome was prevailing in 188 (42.9\%) population. NAFLD is strongly associated with insulin resistance (IR) and other components of the metabolic syndrome, like T2DM, central obesity, hyperlipidemia, and hypertension ${ }^{33}$. The pathogenesis of NASH appears to be a multiple hit process. The initial insult is the development of macrovesicular steatosis with the accumulation of hepatic fat from decreased hepatic free fatty acid oxidation and D or increased hepatic de novo lipogenesis, and D or decreased lipid export from the liver. Although IR can contribute to this dysregulation of lipid metabolism, once fatty liver develops, it can worsen hepatic IR and diabetes, contributing to a vicious cycle $^{34}$.

Serum ALT and AST levels were similar in NASH and NNFL in this study. But GGT were significantly $(\mathrm{P}=$ 0.05 ) higher in NASH than that of NNFL. NASH has been associated with slight elevation of liver enzymes mostly $\mathrm{ALT}^{35}$. In other reports NAFLD patient typically present with asymptomatic serum aminotransferase elevations of 2-3 times the normal ${ }^{36}$. This difference was due to different selection criteria. GGT is a sensitive indicator of liver damage ${ }^{37}$. Excess deposition of fat in the liver is associated with an elevated serum GGT ${ }^{38}$. Recent reports suggest that an increased GGT level is a risk factor for advanced fibrosis in NAFLD and, with weight loss, a decrease in GGT activity is predictive of improved lobular inflammation and fibrosis of liver $^{39}$. 
The limitation of the study was that we had not done it at the community level rather at a tertiary level hospital of the country.

In conclusion, Females were predominant sufferer of NAFLD in Bangladesh. Prevalence of NASH was much higher in NAFLD. Diabetes was the main culprit in developing NASH in NAFLD. GGT was the only biochemical predictor of NASH but with low sensitivity and specificity. We recommend liver biopsy in NAFLD with diabetes and raised GGT.

\section{References:}

1 Ludwig J, Viggiano TR, McGill DB, Oh BJ.Nonalcoholic steatohepatitis: mayo Clinic experience with a hitherto unnamed disease. Mayo Clin Proc1980; 55: 434-38.

2. Browning JD, Szczepaniak LS, Dobbins R, Nuremberg P, Horton JD, Cohen JC, et al. Prevalence of hepatic steatosis in an urban population in the United States: impact of ethnicity. Hepatology 2004; 40: 1387-95

3. Angulo P. GI epidemiology: nonalcoholic fatty liver disease. Aliment PharmacolTher 2007; 25: 883-9.

4. Matteoni CA, Younossi ZM, GramlichT, Boparai N, Liu YC, McCullough AJ.Nonalcoholic fatty liver disease: a spectrum of clinical and pathological severity. Gastroenterology1999; 116:1413.

5. Misra A, Chowbey P, Makkar BM, Vikram NK, Wasir JS, Chadha D, et al. For the Consensus Group. Consensus statement for diagnosis of obesity, abdominal obesity and the metabolic syndrome for Asian Indians and recommendations for physical activity, medical and surgical management. J Assoc Physicians India 2009; 57: 163-70.

6. Amarapurkar D, Kamani P, Patel N, Gupte P, Kumar P, Agal $\mathrm{S}$, et al. Prevalence of non-alcoholic fatty liver disease: population based study. Ann Hepatol. 2007;6:161.

7. Grattagliano I, Portincasa P, Palmieri VO, Palasciano G.Managing nonalcoholic fatty liver disease: recommendations for family physicians. Can Fam Physician 2007; 53: 857-63.

8. Fan JG, Farrell GC. Epidemiology of non-alcoholic fatty liver disease in China. J Hepatol 2009; 50: 204-10.

9. Marchesini G, Bugianesi E, Forlani G, Cerrelli F, Lenzi M, Manini R, et al. Nonalcoholic fatty liver, steatohepatitis, and the metabolic syndrome. Hepatology 2003; 37: 917-923.

10. Angulo P, Keach JC, Batts KP, Lindor KD.Independent predictors of liver fibrosis in patients with nonalcoholic steatohepatitis. Hepatology 1999; 30:1356-62.

11. Marchesini G, Brizi M, Bianchi G, Tomassetti S, Bugianesi E, Lenzi M,et al. Nonalcoholic fatty liver disease: a feature of the metabolic syndrome. Diabetes2001; 50:1844-1850.
12. Argo CK, Northup PG, Al-Osaimi AM, Caldwell SH. Systematic review of risk factors for fibrosis progression in non-alcoholic steatohepatitis. J. Hepatol. 2009; 51:371 - 379.

13. Powell EE, Cooksley WG, Hanson R. The natural history of nonalcoholic steatohepatitis: a follow-up study of forty - two patients for up to 21 years. Hepatology 1990; 11: 74 - 80.

14. Hui JM, Kench JG,Chitturi S. Long-term outcomes of cirrhosis in nonalcoholic steatohepatitis compared with hepatitis C. Hepatology 2003; 38: 420-427.

15. Chitturi S, Farrell GC, Hashimoto E, Saibara T, Lau GK, Sollano JD. The Asia-Pacific Working Party on NAFLD. Nonalcoholic fatty liver disease in the Asia-Pacific region: Definitions and overview of proposed guidelines. J GastroenterolHepatol. 2007; 22:778-87.

16. Brunt EM, Janney CG, Di Bisceglie AM, Neuschwander-Tetri BA, Bacon BR. Nonalcoholic steatohepatitis: a proposal for grading and staging the histological lesions. Am J Gastroenterol 1999; 94:2467-74.

17. Kleiner DE, Brunt EM, Van Natta M, Behling C, Contos MJ, Cummings OW, et al. Design and validation of a histological scoring system for nonalcoholic fatty liver disease. Hepatology 2005; 41:1313-1321.

18. Annurad E, Shiwaku K, Nogi A, Kitajima K, Enkhmaa B, Shimono K, et al. The New BMI criteria for Asians by the Regional Office for the Western Pacific Region of WHO are suitable for the screening overweight to prevent metabolic syndrome in Elder Japanese Workers. J occup Health 2003; 45: 335-343.

19. Li H, Wang YJ, Tan K, Zeng L, Liu L, Liu FJ, et al. Prevalence and risk factors of fatty liver disease in Chengdu, Southwest China. HepatobiliaryPancreat Dis Int. 2009;8:377-82.

20. Park SH, Jeon WK, Kim SH, Kim HJ, Park DI, Cho YK, et al. Prevalence and risk factors of non-alcoholic fatty liver disease among Korean adults. J GastroenterolHepatol. 2006;21:138-43.

21. Vernon G, Baranova A, Younossi ZM. Systematic review: the epidemiology and natural history of non-alcoholic fatty liver disease and nonalcoholic steatohepatitis in adults. Aliment Pharmacol Ther 2011; 34: 274-85.

22. Adams LA, Lymp JF, St Sauver J, Sanderson SO, Lindor KD, Feldstein A, et al. The natural history of nonalcoholic fatty liver disease: a population-based cohort study. Gastroenterology. 2005;129: 113-21.

23. Mohan V, Farooq S, Deepa M, Ravikumar R, Pitchumoni CS. Prevalence of non-alcoholic fatty liver disease in urban south Indians in relation to different grades of glucose intolerance and metabolic syndrome. Diabetes Res ClinPract. 2009; 84:84-91.

24. Hamaguchi M, Takeda N, Kojima T, Ohbora A, Kato T, Sarui $\mathrm{H}$, et al. Identification of individuals with non-alcoholic fatty liver disease by the diagnostic criteria for the metabolic syndrome. World J Gastroenterol 2012; 18:1508-1516. 
25. Manco M, Bedogni G, Marcellini M, Devito R, Ciampalini P, Sartorelli MR, et al. Waist circumference correlates with liver fibrosis in children with nonalcoholic steatohepatitis. Gut. 2008;57: 1283-87.

26. Sato K,Dohke M, Mizutome N, Kimura R, Makuri A, Matsui A, et al. Evaluation of metabolic syndrome with respect to the waist circumference and visceral fat area. Ningen Dock. 2008;23:558-563.

27. Neuschwander-Tetri BA.Nontriglyceride hepatic lipotoxicity: the new paradigm for the pathogenesis of NASH. Curr Gastroenterol Rep 2010;12:49-56.

28. Neuschwander-Tetri BA. Hepatic lipotoxicity and the pathogenesis of nonalcoholic steatohepatitis: the central role of nontriglyceride fatty acid metabolites. Hepatology 2010;52:774-88.

29. Farrell GC, RooyenDv, Gan L, Chitturi S. NASH is an Inflammatory Disorder: Pathogenic, Prognostic and Therapeutic Implications. Gut Liver 2012;6:149-171.

30. McCullough AJ. The epidemiology and risk factors of NASH. In:FarrellGC,GeorgeJ,de la Hall P, et al., eds. Fatty Liver Disease: NASH and Related Disorders. Malden, MA: Blackwell Publishing; 2005:23-37.

31. Farrell GC, Larter CZ. Nonalcohlic fatty liver disease: form steatosis to cirrhosis. Hepatology 2006; 43: s99-s112.
32. Ratziu V, Poynard T. Assesing the outcome of nonalcohlic steatohepatitis? It's time to get serious. Hepatology 2006; 44:802-5

33. Clark JM, Brancati FL, Diehl AM. The prevalence and etiology of elevated aminotransferase levels in the united states. Am J Gastroenterol 2003; 98: 960-67.

34. Edmison J, McCullough AJ. Pathogenesis of non-alcoholic steatohepatitis: human data. Clin Liver Dis 2007; 11: 75-104.

35. Bayard M, Holt J, Boroughs E. Nonalcoholic fatty liver disease. Am Fam Physician2006; 73: 1961-8.

36. Marceau P, Biron S, Hould FS, Marceau S, Simard S, Thung $\mathrm{SN}$, et al. Liver pathology and the metabolic syndrome $\mathrm{X}$ in severe obesity. J ClinEndocrinolMetab 1999;. 84: 1513-7.

37. Lee DH, Blomhoff R, Jacobs DR Jr. Is serum gamma glutamyltransferase a marker of oxidative stress? Free Radic Res 2004; 38: 535-9.

38. Saito T, Nishise Y, Makino N, Haga H, Ishii R, Okumoto K, et al. Impact of metabolic syndrome on elevated serum alanine aminotransferase levels in the Japanese population. Metabolism 2009; 58: 1067-75.

39. Kim HC, NamC, Jee SH, Han KH, Oh DK, Suh I. Normal serum aminotransferase concentration and risk of mortality from liver diseases: prospective cohort study. BMJ 2004; 328: 983. 